\title{
The Beneficial Effects of the Buttonhole Technique Used for the Cannulation of Arteriovenous Fistula Among Hemodialysis Patients: A Literature Review
}

\author{
Haihui Yin ${ }^{1,}$, Caiping Zhou ${ }^{1}$, Haiyan Yu', Wei Ren ${ }^{2}$ \\ ${ }^{1}$ Blood Purification Center of the 1st Affiliated Hospital, University of Science and Technology of China, Hefei, P.R.China \\ ${ }^{2}$ Department of Nephrology of the 1st Affiliated Hospital, University of Science and Technology of China, Hefei, P.R.China
}

Email address:

08901054r@connect.polyu.hk (Haihui Yin)

*Corresponding author

\section{To cite this article:}

Haihui Yin, Caiping Zhou, Haiyan Yu, Wei Ren. The Beneficial Effects of the Buttonhole Technique Used for The Cannulation of Arteriovenous Fistula Among Hemodialysis Patients: A Literature Review. American Journal of Nursing Science.

Vol. 10, No. 3, 2020, pp. 185-190. doi: 10.11648/j.ajns.20211003.19

Received: April 5, 2020; Accepted: June 24, 2021; Published: June 29, 2021

\begin{abstract}
Arteriovenous fistula (AVF) is a "life-line" for hemodialysis patients. Complications associated with AVF cannulation including the occurrence of vessel stenosis and formation of false aneurysms can substantially shorten the length of AVF survival and its utilization. To minimize the damages to vessel walls of AVF, various needling techniques involving the utilization of sharp or blunt needles, and rotating puncture sites are applied in clinical practice. The buttonhole cannulation (BHC) method appears to be a successful technique to solve the problem of puncturing difficult AVFs. However, it is controversial whether BHC should be extended widely to actual practice to generate further beneficial effects or be restricted in clinical use due to a higher risk of adverse AVF complications. This review reports and elaborates on the methods used to create BHC tunnels, the effects of BHC on patient outcomes compared with other cannulation techniques with regard to both objective and subjective aspects, the advantages and disadvantages of $\mathrm{BHC}$, and the measures for improving BHC. According to the published literature, utilizing BHC for AVF cannulation can effectively prevent the complications associated with AVF, while formulating and implementing the standardized BHC procedure, together with comprehensive staff training and selfmanagement education for patients, can substantially reduce the risk of infection. Future studies with rigorous design need to investigate the long-term effects and strengthen the existing evidence regarding the utilization of BHC.
\end{abstract}

Keywords: Button-hole Technique (BHC), Arteriovenous Fistula (AVF), Complications, Hemodialysis

\section{Introduction}

Arteriovenous fistula (AVF) is a "life-line" for hemodialysis patients. Traditional cannulation methods, such as the utilization of sharp needles and frequent changes of needling sites and puncturing angles, can inevitably cause massive damage to the walls of a vessel. This can lead to vascular stenosis or to the formation of false aneurysms, which would substantially shorten the length of AVF survival and its utilization. According to the practice guidelines published by the National Kidney Foundation (NKF, 2006), buttonhole puncture cannulation (BHC) is the optimal technique for minimizing AVF complications [1]. However, some studies have reported that $\mathrm{BHC}$ is associated with a higher risk of local or systemic infections, and have recommended that its extensive application among hemodialysis patients should be restricted [2-3]. Other studies have found that $\mathrm{BHC}$ was not necessarily associated with more infection events nor did it have a negative impact on the function of AVF [4-5]. The purpose of this review was to summarize the various current applications of $\mathrm{BHC}$, the advantages and disadvantages of $\mathrm{BHC}$, and the measures that have been made to improve BHC.

\section{Commonly Used Techniques for AVF Cannulation}

There are currently three methods of AVF cannulation that are 
widely used in clinical areas around the world. The area puncture method (AP), the oldest method, involves using sharp needles to repeatedly puncture a small area. The major disadvantage of this method is that it can cause massive damage to the walls of a vessel, leading to the formation of an aneurysm and to further stenosis. The procedure is also painful. In the rope ladder method (RL) sharp needles are also used to puncture the wall of the vessel from the bottom to the upper side. Puncture sites are shifted regularly, making this method superior to AP in protecting AVF vessels. The obvious disadvantage of RL is that the AVF vessel must be of an adequate length for cannulation. BHC originated from the "Constant Site Method," which was initiated by Twardowski and colleagues at the end of the 1970's [6]. The key feature of the "Constant Site Method" is that the exact same tunnel is used for cannulation, with the consequent formation of a simple, painless tunnel to the wall of the AVF vessel. Initially, the intention was to solve the problem of AVF vessels being of limited length for cannulation, since RL could hardly be applied to patients with such vessels. This cannulation technique was renamed BHC by Kronung [7] in 1984. BHC is comprised of two stages: establishing the BHC tunnel, and then using blunt needles for cannulation.

\section{Methods to Create BHC Tunnels}

BHC has been widely applied in Japan and European countries for more than three decades, and is also used in the U.S.A. and Canada [8]. In the past few years, it has also come into use in mainland China [9-12]. At present, there are three commonly used methods to create BHC tunnels in clinical settings: the CS method, the BH Stick method, and the use of a dialysis needle (Clampcath $\left.{ }^{\circledR}\right)$ [13].

\subsection{Constant Site Method (CS)}

The CS method is the oldest and the primary method for creating BHC tunnels in clinical areas. The CS method includes the following steps: one specific nurse uses a sharp needle to puncture a patient 6 -12 times at the same angle and depth, to form BHC tunnels $[9,14]$. At the maintenance stage blunt needles can be used and the puncture procedure can also be performed by other nurses [15]. Essential to the CS method are the three "SAMEs," referring to the same nurse, the same angle, and the same depth for puncturing, with the latter two aspects also being crucial to the forming of $\mathrm{BHC}$ tunnels. Even after the formation of the BHC tunnels, for successful cannulation with blunt needles those who later perform the procedure need to follow the exact angle and depth as those used by the initial creator of the tunnels. Although international researchers have reported that two or three experienced nurses can create the same BHC tunnel [16-17], the major drawback to extending the CS method in clinical practice is still the requirement that ONE specific nurse preside over the creation of the BHC tunnels [8].

\subsection{BH Stick}

Japanese researcher Toma was the first person to use a BH
Stick (manufactured by Nipro Medical Ltd., Japan) to create BHC tunnels [18]. The procedure is as follows. After the completion of each hemodialysis treatment session, the $\mathrm{BH}$ Stick is inserted in the puncture site immediately after the dialysis needle is removed and hemostasis achieved. The above procedure needs to be repeated for 2 weeks to form BHC tunnels. There are some advantages to using BH Sticks to create BHC tunnels: the procedure is easy to perform, the sticks are practical to use, and no specialized operators are required to perform this procedure. Compared with the CS method, using BH Sticks can significantly reduce the severity of a patient's pain and improve puncture success rates [19]. Patients have also reported that there was no interference with their daily activities even during the period when the BH Stick was left in situ [18]. BH Sticks are widely accepted and welcomed by nurses and patients in the United Kingdom [19] and Canada [20]. The only disadvantage to using $\mathrm{BH}$ Sticks is the cost associated with the procedure, because at least 6 pairs of BH Sticks are needed to create BHC tunnels (BioHole ${ }^{\mathrm{TM}}$, produced by $3 \mathrm{M}$ Medical Ltd., U.S.A; or BH Stick, produced by Nipro Medical Ltd., Japan), at a cost of approximately 60 Euros for each patient [19].

\subsection{Using a Dialysis Needle (Clampcath $囚)$}

Marticorena et al. reported that a dialysis needle (Clampcath SP $502^{\circledR}, 17 \mathrm{G}$, produced by Togo Medikit Ltd., Japan) was used to establish arteriovenous access, and was then left in situ for 10 days to create BHC tunnels [21]. This method included several steps: first, the optimal puncture site was selected using bed site ultrasound; then, Clampcath ${ }^{\circledR}$ was used to establish vascular access for the hemodialysis treatment. After each treatment session, the endovascular polyurethane catheters were flushed using $0.9 \%$ normal saline, and were then locked by heparinised saline with sterile connectors attached. The limb on which the puncture site is located needs to be carefully fixed with a splint and elastic bandages. On the $10^{\text {th }}$ day, the two endovascular catheters can be removed after a dialysis session, and blunt needles (Nipro 15G, produced by Nipro Medical Ltd., Japan) can be used for cannulation if BHC tunnels have been successfully created [21]. The major advantage of this method is that only one puncture is required during the period that the BHC tunnels are being created, and no specialized personnel are required to perform this procedure. In addition, this approach is easy to perform and practical to use in actual practice. However, the potential risks during the period that the catheter remains in situ should be considered. These include catheter blockage, accidental breakage or removal, local bleeding, and infections.

\section{The Clinical Effects of BHC Compared with Other Cannulation Methods}

There are studies comparing both the subjective and objective outcomes of using BHC and other methods of AVF 
cannulation. The majority of the studies indicated that BHC can effectively reduce the severity of the patients' pain during the cannulation procedure, the occurrence of AVF complications, and the workload for health professionals, especially nursing staff. However, some researchers have emphasized that the increased risk of infection associated with the utilization of BHC should be carefully reviewed in clinical practice.

\subsection{Patients' Subjective Experience}

Most researchers have reported that patients in the $\mathrm{BHC}$ group experienced significantly less pain than patients using other methods of cannulation, for the following reasons. During the process of forming BHC tunnels, scar tissues are circled, which might greatly reduce the patient's sensitivity to the perception of pain. In addition, using blunt needles for cannulation avoids the damage to subcutaneous tissue caused by sharp needles, thus effectively alleviating the pain experienced by patients. In Japan, Toma and colleagues reported that pain scores had dropped significantly after BHC was used in one group in a pre and post-test design study involving 37 patients [18]. In Canada, Marticorena and team [22] conducted a prospective study to assess the pain severity of patients who were observed for 1 year. They found that the pain severity at the 6th month was significantly lower than that at the 2nd week after BHC was used for cannulation. Patients who received hemodialysis daily also experienced less pain than patients who received hemodialysis every other day (three times a week) [22]. Ludlow [23] also reported that pain sores were significantly lower in the BHC group than in other groups during a 3-month follow-up period. In the UK, a randomized controlled trial (RCT) conducted by Struthers and colleagues [16] revealed that the patients' pain scores dropped significantly during the 6-month observation period, and that the requirement for local analgesia was also reduced ( 9 cases in the BHC group did not use local tropical analgesia vs. 1 case in the RL group). In Australia, Hartig et al. [24] conducted a survey of patients in 6 hemodialysis centers. They found that $69 \%$ of the patients reported that the severity of their pain was reduced after the BHC method was used, and that $63 \%$ reported that their anxiety levels had also dropped. In mainland China, similar findings were reported by Wang et al. [9] and Ma et al. [11] regarding the significant pain relief that occurred during the cannulation procedure compared with that experienced by the AP method group. However, some other researchers have found pain scores to be significantly higher in the BHC group than in the RL method group [17, 25], or found no significant differences between the use of BHC and other methods [15]. Despite the controversial results, hemodialysis patients expressed more confidence and satisfaction with AVF cannulation because the method is less difficult for home dialysis patients to employ when carrying out self-cannulation, and because the cosmetic needs for local area to be met by using BHC method as well [26].

\subsection{Objective Clinical Outcomes}

Most researchers observed more AVF complications such as local bleeding, infection, stenosis, and the formation of false aneurysms associated with the utilization of BHC compared with other puncture methods. The occurrence of stenosis and the formation of AVF aneurysms were significantly lower in the BHC group because only one puncture site was made and damage to vessel walls from cuts was minimized due to the use of blunt needles.

\subsubsection{Local Bleeding}

Toma et al. reported that the occurrence of local bleeding was significantly lower after using $\mathrm{BHC}$ and that the time required for hemostasis after the removal of dialysis needles was less than $10 \mathrm{~min}$ in $95 \%$ of the patients [18]. Similar results were reported by Marticorena and colleagues [22], who found that local bleeding ceased during the dialytic treatment session and that the time required for hemostasis after dialysis fell from 24 min to 15 min within 2 weeks of using BHC for patients. Verhallen et al. [26] also reported that the time required for hemostasis was significantly shorter after using BHC for home dialysis patients. In Chinese patients, similar findings were reported with regard to injection site bleeding, and less time was required for hemostasis compared with other cannulation methods [10-11]. In addition, the occurrence of subcutaneous hematoma was significantly lower in the BHC group than in the RL group [16-17]. No hematoma formation occurred in $87.5 \%$ of patients using BHC in a small study population (14 out of 16) [24]. However, some researchers reported that the occurrence of hematoma was significantly higher in the BHC group than in the RL group [25].

\subsubsection{Long-term AVF Complications}

Positive effects were found after using BHC for cannulation with regard to long-term AVF complications such as vascular stenosis and the formation of false aneurysms. Struthers and colleagues [16] reported that there was no change in the diameter of AVF vessels in the BHC group during the 6-month follow-up period, while an enlargement of nearly $30 \%$ (around $5 \mathrm{~mm}$ ) was found in the RL group. Marticorena et al. [22] also reported beneficial effects for patients with evident AVF aneurysms and thin local subcutaneous tissue after using BHC for 6 months: local skin abnormalities had almost disappeared, and in two cases the AVF aneurysm was significantly smaller than before at the 1-year assessment after using BHC. Similar findings were reported by other researchers, as there was no further dilation of AVF aneurysms in the BHC group [26]. Chinese researchers $[9,11]$ also reported that the occurrence of vascular stenosis and the formation of aneurysms were significantly lower in the BHC group than in other groups ( $13.33 \%$ vs. $100.0 \%$ and $6.67 \%$ vs. $100.0 \%$ respectively) [9].

\subsubsection{Infections}

Previous studies found that AVF cannulation rarely led to infections. Tom and colleagues reported that only one infection occurred among 37 patients using BHC [18]. Other researchers also supported the above findings, determining that no significant increase in infections was associated with 
using BHC [11, 21-22, 26]. However, a few studies have reported that the risk of infection had increased significantly due to some aspects related to the BHC procedure, such as the incomplete removal of scabs on puncture sites, the accidental entry of fragments of scabs into BHC tunnels or vessels, multiple attempts at puncturing leading to false tunnels, or damage to the inner walls of BHC tunnels from the use of sharp needles. All of these factors might provide the room for bacteria to colonize and cause infections. Doss et al. [27] reported that the rate of infection was 0.16 and 0.19 / 1000 patient days for in-center dialysis patients and home dialysis patients respectively after using BHC. Ten cases of sepsis were reported and the results of the blood culture tests were as follows: 3 staphylococcus aureus infections, 1 group B streptococcus infection, and 2 Grampositive cocci infections. In addition, 13 BHC puncture site infections occurred: 10 staphylococcus aureus infections and 3 MRSA (Methicilline-resistant Staphylococcus Aureus, MRSA) infections [27]. In a retrospective study, Nesrallah and colleagues reported that the occurrence of staphylococcus aureus infection and its dissemination was significantly associated with the utilization of BHC among home dialysis patients [28]. The study indicated that 10 out of 56 patients had staphylococcus aureus infections, and 4 cases of dissemination of infection occurred among those patients (including 2 cases of pneumonia, 1 case of bacterial arthritis, and 1 case of epidural abscess). When compared with the RL group, the rate of staphylococcus aureus infections increased 46-fold in the BHC group (0.23 / 1000 AVF days vs. 0.005 / 1000 AVF days) [28]. Labriola et al. [29] also reported that AVF infections increased after the use of BHC from the previous RL method (from 0.31 / 1000 AVF days to 0.43 / 1000 AVF days). In a randomized controlled trial, Chow et al. [25] reported that the rates of puncture site infections were significantly higher in the BHC group than in the RL group (4 / 34 vs. 1/35).

\subsubsection{Difficulties in Cannulation}

Researchers reported that the ease of conducting AVF cannulation improved significantly with the length of time that BHC was used, from 1 month to 1 year [20, 22]. The success rates for cannulation in the BHC group were also significantly higher than those in the other group (98.25\% vs. $83.24 \%$ ) [11]. In addition, anxiety levels among the majority of nurses $(75 \%)$ were lower when performing cannulation for difficult patients, and $83 \%$ of nurses agreed that less time was required for the cannulation procedure when using BHC. The major advantage of $\mathrm{BHC}$ was its ease of use for cannulation [24], and most nursing staff (61\%) were willing to use this technique [16]. However, it was also controversially reported that the failure rates for cannulation in the BHC group were significantly higher than those in the RL group [17].

Galante et al. reported that the failure rates for $\mathrm{BHC}$ (43.7\%) had dropped significantly after the implementation of improvement measures [30]. They also analyzed all related factors and proposed constructive strategies for improvement. These involved adopting single cannulators to establish and maintain BHC tunnels, positioning the patient's limb correctly, utilizing a tourniquet, avoiding violent puncturing, and using the "Touch Cannulation Technique." They also suggested that a standardized cannulation procedure for $\mathrm{BHC}$ be formulated and implemented for actual practice [30]. In addition, pitted puncture sites have been found to be a crucial factor contributing to difficult cannulation and to have led to the incomplete removal of scabs, the disinfection of local skin, and to the aggravation of injuries to the entrance and inner wall of BHC tunnels [31].

\subsubsection{Medical Costs Associated with BHC}

Ludlow analyzed the cost of using BHC for hemodialysis patients and found no significant difference between the $\mathrm{BHC}$ and other groups [23]. However, some researchers have pointed that the medical materials required to carry out the $\mathrm{BHC}$ procedure, such as blunt needles, forceps for removing scabs, gauze or cotton balls for soaking scabs, and BH sticks for creating BHC tunnels, might increase the medical expenses of patients [18].

\section{Strategies for Improving BHC}

Two major strategies associated with a reduction in AVF infections and failure rates for cannulation have been recommended as measures to improve BHC.

\subsection{To Prevent AVF Infections}

Most researchers agreed that a systematic quality improvement program that includes implementing a standardized BHC cannulation procedure, providing comprehensive staff training, and self-management for patients could effectively reduce the risk of infections associated with BHC. Birchenough and colleagues [32] conducted a retrospective study to evaluate the effectiveness of a quality improvement program on AVF infections for patients using BHC. The results indicated that the rate of AVF infection among the BHC group was 52.0\% (4/7.7 patient year), which was more than 10 -fold that in the other group $(4.9 \%, 2 / 40.7$ patient year). They carried out a comprehensive quality improvement program in their study unit. The program covered key aspects, such as the formulation of a standardized procedure for BHC cannulation (including details of the process for creating BHC tunnels, the BHC cannulation procedure, and techniques for trouble shooting), patient education in the knowledge and skills required for self-management with $\mathrm{BHC}$, together with a meticulous assessment of the puncture site and the patients' adherence by using a specially designed form before conducting cannulation. The infection rate fell to $29.8 \%$ (2/6.7 patient year) 14 months after the implementation of the above quality improvement measures [32]. The results of the study by Labriola et al. [29] also supported the view that comprehensive staff training and strict adherence to a standardized procedure for $\mathrm{BHC}$ in actual practice correlates 
to a reduction in AVF infections (0.43/1000 AVF days before training vs. 0.34 / 1000 AVF days after training); and an infection rate in the BHC group that was similar to that in the RL group (0.31 / 1000 AVF days). Ball [14] suggested that appropriate preparation of the BHC puncture site was one of the key strategies for reducing the occurrence of AVF infections. The following aspects should be involved in the preparation procedure: thorough cleansing and disinfecting of the skin around the puncture site, complete removal of scabs, keeping sterile dressings on puncture site covered during the treatment session, and the topical use of Mupirocin ointment [14]. Nesrallah et al. [28] reported that topical use of Mupirocin ointment for the BHC puncture site can effectively prevent Staphylococcus Aureus infections. According to the results of the study, no Staphylococcus Aureus infections occurred in 56 patients with the prophylactic use of Mupirocin on the puncture site, while 10 cases of positive blood cultures with Staphylococcus Aureus were indicated among those patients before antibiotic ointment was used as a preventive measure [28].

\subsection{Solving the Difficulties of Cannulation}

Key points to solving the difficulties involved in cannulation include the successful creation of BHC tunnels and the careful maintenance of them, together with the application of correct cannulation techniques for BHC. Ball [8] suggested that the optimal choice was to adopt the ONE-nurse method in the creation of BHC tunnels, and then to record the angle for cannulation, using the same angle for cannulation to access the vascular wall to minimize the occurrence of tunnel-related complications. The advantage of the ONE-nurse method for the creation and maintenance of BHC tunnels is the consistency in the diameter of the tunnels and in the use of dialysis needles, consequently minimizing the need to make multiple attempts at puncturing from multiple angles. This reduces the injuries that are made to the inner walls of BHC tunnels and prevents tunnel infections caused by multiple false tunnels or by leaving space for bacteria to survive. Blunt needles should be used for cannulation to minimize the injuries made to the inner walls of BHC tunnels immediately after the tunnels are successfully created using 8 to 10 punctures from sharp needles.

The correct technique for $\mathrm{BHC}$ cannulation is to align the puncture site, the BHC tunnel, and the access to the vascular walls, to make it easier for blunt needles to pass through the tunnels and open up the vascular valves $[8,14]$. The essential points are to use the same angles as those used by the creator of the BHC tunnel, employ the "Touching Cannulation Technique," properly position the patient's upper limb, use tourniquets, and educate patients about the need to avoid the excessive intake of fluids to prevent the vascular access from shifting. In addition, the skin around the puncture site should be pulled tightly to prevent the vessel from sliding during the cannulation procedure. The needle can be pulled out slightly and the puncturing angles adjusted to search for the vascular access if resistance is encountered, while violent puncturing or the use of sharp needles should be avoided. Further, the needle should not be fully inserted into the skin; rather, approximately $2 \mathrm{~mm}$ of it needs to remain outside to prevent pitting at the site of the puncture, future difficulties with removing scabs and with cannulation, tunnel damage, and the occurrence of infections [31].

\section{Conclusions}

In summary, BHC is effective at alleviating the painful experience of cannulation for patients, reducing the work load of health professionals, reducing the complications associated with AVF, and extending its survival time. Effective preventative measures to minimize the risk of infection include the formulation of a standardized $\mathrm{BHC}$ procedure, comprehensive staff training in related knowledge and skills, and patient education for self-management. Over the long term, BHC might reduce the medical expenses of patients and the utilization of health resources associated with AVF complications. Thus, it can be applied and extended to clinical practice. Future studies with rigorous design need to investigate the long-term effects and strengthen the existing evidence regarding the utilization of BHC.

\section{References}

[1] NKF-KDOQI. Clinical practice guidelines for vascular access: update July 2006. Am J Kidney Dis, 2006, 48 (Suppl 1): S187-S277.

[2] Glerup R, Svensson M, Jensen JD, et al. Staphylococcus aureus bacteremia risk in hemodialysis patients using the buttonhole cannualtion technique: a prospective multicenter study. Kidney Med, 2019, 1 (5): 263-270.

[3] Lyman M, Nguyen DB, Shugart A, et al. Risk of vascular access infection associated with buttonhole cannulation of fistulas: data from the National Healthcare Safety Network. Am J Kidney Dis, 2020, 76 (1): 82-89.

[4] Morgans HA, De Souza HG, Richardson T, et al. A comparison of the buttonhole and rope-ladder AVF cannulation techniques and infection rates within the SCOPE collaborative. Pediatr Nephrol, 2021 Jun 11. doi: 10.1007/s00467-021-05137-1.

[5] Zhao HZ, Huang HP, Fang SY, et al. Application of blunt needle buttonhole technique in arteriovenous fistula puncture for patients underwent hemodialysis [in Chinese]. Nurs J Chin PLA, 2016, 33 (8): 65-67.

[6] Twardowski Z, Kubara H. Different sites versus constant sites of needle insertion into arteriovenous fistula for treatment by repeated hemodialysis. Dial Transplant, 1979, 8 (10): 978-980.

[7] Kronung G. Plastic deformation of Cimino fistula by repeated puncture. Dial Transplant, 1984, 13 (10): 635-638.

[8] Ball LK. The buttonhole technique for arteriovenous fistula cannulation. Nephrol Nurs J, 2006, 33 (3): 299-304.

[9] Wang WJ, Wu YC, Ying YJ, et al. The impacts of puncture methods on arteriovenous fistula for hemodialysis [in Chinese]. Chin J Nurs, 2009, 44 (2): 180-181. 
[10] Wang WJ, Ying YJ, Wu CY, et al. Effects of using blunt needles for buttonhole cannulation on arteriovenous fistula complications for hemodialysis patients [in Chinese]. Chin Pract J Nurs, 2010, 26 (9B): 26-27.

[11] Ma LL, Mei XR, Wang LP, et al. Application and management of buttonhole puncture technique for hemodialysis patients with difficult arteriovenous fistula cannulation [in Chinese]. Chin Integ J of TCM \& West Med, 2012, 13 (5): 442-443.

[12] Hu MJ. Application and management for buttonhole technique for arteriovenous fistulae cannulation [in Chinese]. Chin Prim Med, 2012, 19 (1): 147-148.

[13] Kumbar L. Complications of arteriovenous fistulae: beyond venous stenosis. Adv Chronic Kidney Dis, 2012, 19 (3): 195-201.

[14] Ball LK. The buttonhole technique: strategies to reduce infections. Nephrol Nurs J, 2010, 37 (5): 473-478.

[15] Castro MC, Silva Cde F, Souza JM, et al. Arteriovenous fistula cannulation by buttonhole technique using dull needle. J Bras Nefrol, 2010, 32 (3): 281-285.

[16] Struthers J, Allan A, Peel RK, et al. Buttonhole needling of ateriovenous fistulae: a randomized controlled trial. ASAIO J, 2010, 56 (4): 319-322.

[17] van Loon MM, Goovaerts T, Kessels AG, et al. Buttonhole needling of haemodialysis arteriovenous fistulae results in less complications and interventions compared to the rope-ladder technique. Nephrol Dial Transplant, 2010, 25 (1): 225-230.

[18] Toma S, Shinzato T, Fukui H, et al. A timesaving method to create a fixed puncture route for the buttonhole technique. Nephrol Dial Transplant, 2003, 18 (10): 2118-2121.

[19] King J. Implementing the buttonhole method using the Biohole peg in a busy dialysis unit: a report of the development of current practice. J Ren Care, 2009, 35 (4): 192-200.

[20] Marticorena RM, Hunter J, Macleod S, et al. Use of the BioHole ${ }^{\mathrm{TM}}$ device for the creation of tunnel tracks for buttonhole cannulation of fistula for hemodialysis. Hemodial Int., 2011, 15 (2): 243-249.

[21] Marticorena RM, Hunter J, Cook R, et al. A simple method to create buttonhole cannulation tracks in a busy hemodialysis unit. Hemodial Int, 2009, 13 (3): 316-321.

[22] Marticorena RM, Hunter J, Macleod S, et al. The salvage of aneurysmal fistulae utilizing a modified buttonhole cannulation technique and multiple cannulators. Hemodial Int, 2006, 10 (2): 193-200.

[23] Ludlow V. Buttonhole cannulation in hemodialysis: improved outcomes and increased expense--is it worth it? CANNT J, 2010, 20 (1): 29-37.

[24] Hartig V, Smyth W. "Everyone should buttonhole": a novel technique for a regional Australian renal service. J Ren Care, 2009, 35 (3): 114-119.

[25] Chow J, Rayment G, San Miguel S, et al. A randomized controlled trial of buttonhole cannulation for the prevention of fistula access complications. J Ren Care, 2011, 37 (2): 85-93.

[26] Verhallen AM, Kooistra MP, van Jaarsveld BC. Cannulating in haemodialysis: rope-ladder or buttonhole technique? Nephrol Dial Transplant, 2007, 22 (9): 2601-2604.

[27] Doss S, Schiller B, Moran, J. Buttonhole cannulation-An unexpected outcome. Nephrol Nurs J, 2008, 35 (4), 417-419.

[28] Nesrallah GE, Cuerden M, Wong JHS, et al. Staphylococcus aureus bacteremia and buttonhole cannulation: Long-term safety and efficacy of mupirocin prophylaxis. Clin J Am Soc Nephrol, 2010, 5 (6): 1047-1053.

[29] Labriola L, Crott R, Desmet C, et al. Infectious complications following conversion to buttonhole cannulation of native arteriovenous fistulas: a quality improvement report. Am J Kidney Dis, 2011, 57 (3): 442-448.

[30] Galante NZ, Rabelo LL, Yamamoto A, et al. One unit's experiences when establishing buttonhole technique, analysis of reasons for failure of procedure: a report. J Ren Care, 2010, $36(2): 73-80$.

[31] Ball LK, Mott, S. How do you prevent indented buttonhole sites? Nephrol Nurs J, 2010, 37 (4): 427-428, 431.

[32] Birchenough E, Moore C, Stevens K, et al. Buttonhole cannulation in adult patients on hemodialysis: an increased risk of infection? Nephrol Nurs J, 2010, 37 (5): 491-498, 555. 